\title{
Liberal political equality implies proportional representation
}

\author{
Eliora van der Hout • Anthony J. McGann
}

Received: 31 January 2006 / Accepted: 20 February 2009 / Published online: 31 March 2009

(C) The Author(s) 2009. This article is published with open access at Springerlink.com

\begin{abstract}
This article shows that for a single-vote electoral system for a representative body to treat all voters and all parties equally, it must produce results essentially identical to list proportional representation (PR). Democratic theory has often been agnostic concerning representative institutions. Different institutions have been compared in terms of behavioral outcomes rather than axiomatic properties. Building on van der Hout (Annual meeting of the Public Choice Society, San Diego, 2002) result, we show that for an electoral system to completely respect the principle of liberal political equality and popular sovereignty, its results must be equivalent to those of list PR.
\end{abstract}

\section{Introduction}

This article provides an axiomatic justification of proportional representation (PR), similar to May's (1952) theorem for majority rule. It shows that the any single-vote seat allocation rule that treats all voters equally must produce results essentially equivalent to pure PR. This result has considerable normative significance for the electoral systems literature. Previously, it has been claimed that proportionality cannot be justified in terms of the theory of voting. Indeed proportionality has often been proposed as an end in itself for electoral systems, as though it is intrinsically just or fair. Attempts to

E. van der Hout

Universiteit van Tilburg, Room P 3224, P.O. Box 90153, 5000 LE Tilburg, The Netherlands

\section{A. J. McGann ( $\square)$}

Department of Government, University of Essex, Wivenhoe Park, Colchester CO4 3SQ, UK e-mail: amccgann@essex.ac.uk

\section{A. J. McGann}

Department of Political Science, University of California, Irvine, CA 92697, USA 
justify PR have tended to be based either on its intuitive fairness to political parties, or on its being fair to social groups. In contrast, our result shows that proportionality is implied by liberal political equality (the requirement that all individual voters be treated equally) and popular sovereignty (the requirement that the voters alone decide the outcome).

We show that any single-vote seat allocation rule that satisfies liberal political equality and nonnegative responsiveness must produce results essentially identical to those of list PR. We operationalize liberal political equality and popular sovereignty as the qualities of anonymity (the rule does not discriminate between voters on the basis of their identity) and neutrality (the rule does not discriminate between candidates or lists of candidates on the basis of their identities). Nonnegative responsiveness is the requirement that if a list wins extra votes and everything else remains constant, it cannot lose seat share. These qualities are applied not just to the choice between lists, but also between coalitions. This is crucial, because political power is not distributed on the basis of the relative size of lists or parties, but on the relative size of coalitions - or more precisely in terms of which coalitions have a majority of seats and are thus able to elect a government or pass legislation. What we demonstrate is that any anonymous, neutral and nonnegativity responsive seat allocation rule produces a set of majority coalitions identical to that produced by pure PR, except that a coalition that wins over $50 \%$ of vote may sometimes get only exactly $50 \%$ of the seats. If we impose the stronger requirement of positive responsiveness (if a list wins more votes, it must increase its seat share), then the seat share allocation rule must produce a set of wining coalitions absolutely identical to that produced by pure PR. Thus the balance of political power - the relative size of lists and coalitions, and the resulting governing coalition formation game-must be the same as that under pure PR.

This justification of PR, based on the equal treatment of individual voters, is very different to those prevalent in the literature. McLean (1991), while normatively sympathetic to PR, argues that it has no basis in the theory of voting. Lijphart (1994) concludes that for many PR supporters, proportionality is simply a goal in itself, "virtually synonymous with political justice" (p.140). Justifications of the principle of proportionality tend to be in terms of fairness to political parties or social groups, or in terms of the desirable instrumental effects of PR. For example, McLean argues that the case for PR rests on the idea that a legislature should be a microcosm of the population. Similarly, Pitkin (1967) identifies the case for PR in terms of "descriptive representation" (the fair representation of every salient group), while Still (1981) uses the similar concept of "group representation". The political electoral reform discourse frequently emphasizes the unfairness of non-proportionality to certain political parties, who win far fewer seats than their vote share would entitle them to under proportionality (see e.g., Independent Commission on the Voting System 1998). In terms of instrumental justifications, Ward (1995) uses simulations to argue that PR is more likely than any other system to produce policy outcomes close to the median voter, while Powell (2000) makes a similar claim using survey data.

It should be noted that the quality of neutrality does not correspond to the demand that lists or parties should be treated "equally" or "fairly" as this is normally understood. Neutrality is merely the condition that parties are not discriminated on the basis of their identity. This is justified on the basis of popular sovereignty. If all the voters 
who support party A decide instead to support party B, then party B must get all the seats that previously went to party A. Otherwise the allocation of seats would depend on a bias in the decision rule, and not on the decision of the voters, thus violating the principle of popular sovereignty. Neutrality is a minimal condition that virtually all current electoral systems respect-even single-member district plurality satisfies neutrality. Neutrality between coalitions, however, is a stronger quality, and does not follow directly from neutrality between parties or candidates. Neutrality between coalitions means that the electoral system does not discriminate between coalitions, and thus if all the voters supporting coalition A switch to coalition B, then coalition B must get the seats that previously went to coalition A. Although this is a stronger quality than neutrality between parties, it can be justified in terms of popular sovereignty in the same way: if the electoral system allows a group of voters to award a certain number of seats to coalition A but not to coalition B, then a bias in the electoral system is determining the result and not just the preferences of the voters.

The results in this article depend on the use of pure PR. This is a system where the seat share of each list is exactly proportional to the vote share it received. This is, of course, an abstraction, and requires that our results be proved for a seat share allocation rule with infinitely divisible seats, as opposed to a realistic voting system that distributes a finite number of seats. This allows us to separate the problem of what seat share allocation is required by our axioms from the practical problem of how this can be best approximated by a finite seat voting rule (the latter problem is beyond the scope of this article). The reason that it is necessary to use pure PR is that all finite seat PR systems can be made to produce extremely non-proportional results under certain conditions, and thus violate the requirements of our results. Although practical PR systems approximate pure proportionality under most circumstances, if there are a large number of very small parties, the results necessarily diverge from proportionality. For example, if there are 100 seats and a list wins $0.5 \%$, we can either give it a seat (over-representing it by 100\%), or not give it a seat (under-representing it by $100 \%$ ). Suppose we have a large number of such parties. By anonymity and neutrality, we have to treat them all equally. Therefore, we have a large coalition that it either under- or over-represented. From this, it is straightforward to create an example where a coalition gets a majority of seats from a minority of votes. This is precisely what we show is forbidden by anonymity, neutrality and nonnegative responsiveness for coalitions. Thus our results require pure proportionality as an ideal. In practical terms, it is only possible to choose electoral systems that respect this most of the time.

Our results only apply to single-vote electoral systems. There are, of course, seat share allocation rules that allow voters to rank multiple alternatives, single transferable vote being perhaps the most commonly used. Extending our results to these systems is beyond the scope of this article, although we argue in the discussion that our results do place limits on what kind of ordinal vote systems satisfy the requirements of liberal political equality.

Previous technical work on PR has tended to concentrate on the mechanics of seat allocation rules rather than the axiomatic justification of the principle of proportionality (see, e.g., Balinski and Young 1982/2003; Taagepera and Shugart 1988). Austen-Smith and Banks (2005) model the interaction between seat allocation rules and coalition formation. The axiomatic literature has concentrated on social decision 
rules (where a decision is to be made amongst competing alternatives) as opposed to seat allocation rules (where political representation is distributed amongst lists). (see May (1952) on majority rule, Young (1974) and Saari (2003) on the Borda Count and Young (1995) on the Kemeny rule.) van Deemen (1993), however, has shown that certain voting paradoxes apply to list PR systems. Rogowski (1981) suggests that anonymity requires proportionality, but provides no proof. Christiano (1996) demonstrates that single member district plurality elections violate anonymity. Hout et al. (2002) show that the anonymity, neutrality, consistency, faithfulness and topsonlyness imply the plurality ranking property. Our results draw on this insight.

There is considerable axiomatic work on proportionality in contexts other than electoral systems. Examples include the distribution of shared costs within firms (Banker 1981) and the distribution of assets between claimants in cases where the sum of the claims exceeds the assets (Aumann and Maschler 1985; Moulin 2002; O’Neill 1982). In these cases proportionality has an important quality of being strategy-proof. For example, in the case of a bankrupt firm, any non-proportional scheme for distributing the assets creates an incentive for claimants to reallocate their claims amongst themselves. Only proportionality does not discriminate between a large number of small claims and a small number of large claims. We, however, do not view parties as "claimants" with rights to certain seat shares, but rather require that the electoral system respect the equality and sovereignty of the voters.

\section{Results}

We show that liberal political equality (operationalized as the axioms of anonymity and neutrality) implies a single-vote seat allocation rule essentially equivalent to pure PR. First, we show that any single-vote seat share allocation rule that is nonnegativity responsive, neutral and anonymous satisfies the weak plurality ranking property (parties that win more votes get at least an equal number of seats). Then we show that this result applies to coalitions as well as parties (if the seat allocation rule is anonymous and neutral, coalitions whose members win more votes must get at least equal seats in aggregate). In parliaments, governments are typically chosen by majority rule, the vote of investiture usually requiring a coalition of parties. Therefore the outcome depends on the coalition formation game defined by the election and the seat share allocation rule. We show that any seat share allocation rule that is anonymous, neutral and nonnegativity responsive (and thus satisfies the weak plurality ranking property) defines a coalition game identical to that defined by pure PR, except that ties between differently sized coalitions are sometimes possible. If we require positive responsiveness, the resulting coalition game must be identical to that defined by pure PR.

Let us define the set of eligible voters as $N$, with voters numbered $1, \ldots, n$, and the set of alternatives $A$, numbered $1, \ldots, a$. The ballot profile $V$ is a relation on $N \times A$ defined by $(i, j) \in V$, or $i V j$, if and only if $i$ votes for $j$, where $i$ votes for at most one $j$ (abstention is allowed). The function $T$ maps the ballot profile into the total vote for each alternative: $T: V \rightarrow[0, n]^{A}$. The seat share allocation function $E$ maps the ballot profile into the seat share for each alternative: $E: V \rightarrow[0,1]^{A}$. 
We can define the following properties of the seat share function $E$. Let $\sigma$ be a function that permutes $N$. Then $E$ is anonymous iff $\forall V$ and $\sigma, E(V)=E(\sigma V)$. Let $\pi$ be a function that permutes $A$. Then $E$ is neutral iff $\forall V$ and $\pi, \pi E(V)=E(\pi V)$. $E$ satisfies the cancellation property iff $\forall V \forall j$ and $k$ in $A, T_{j}=T_{k} \Rightarrow E_{j}=E_{k}$. (If the vote share for two alternatives are the same, then their seat shares must be the same.) Let $S_{j}(\mathrm{~V})$ be the set of voters that vote for alternative $j$ under ballot profile $V$. Thus $S_{j}(V)=\{i: i V j\}$. Then $E$ is nonnegativity responsive iff $\forall V, V^{\prime}$ in $N \times$ $A$ and $j$ in $A, S_{j}\left(V^{\prime}\right) \supseteq S_{j}(V) \Rightarrow E_{j}\left(V^{\prime}\right) \geq E_{j}(V)$. E satisfies the weak plurality ranking property iff $\forall V \forall j$ and $k$ in $A, T_{j}>T_{k} \Rightarrow E_{j} \geq E_{k}$. (If alternative $j$ wins more votes than alternative $k$, alternative $j$ receives a greater or equal seat share to alternative $k$.)

First, we show that liberal political equality implies the weak plurality ranking property-if party A wins more votes than party B, then it must receive at least equal seat share.

Proposition 1 For any ballot profile $V$, any seat share allocation rule E that is anonymous, neutral and nonnegativity responsive satisfies the weak plurality ranking property. (Proof in Appendix)

The intuition here is straightforward. If two parties have the same number of votes, then by anonymity and neutrality, they must have the same seat share. If in this case one party receives more seats, then either the vote allocation system is inherently biased in its favor (violating neutrality) or some voters' votes count for more than others (violating anonymity). If one party then increases its vote at the expense of the other or by gaining the votes of people who previously abstained, by nonnegative responsiveness it must receive a seat share at least equal to that of the other party. Therefore, if one party receives more votes than another it must receive at least equal seats (the weak plurality ranking property).

We can extend Proposition 1 to apply not just to individual parties, but to coalitions of parties. In multi-party systems, governments are typically not single-party, but are composed of coalitions. Therefore the relative size of various coalitions is as important as the relative size of parties. We may arbitrarily partition the set of alternatives $A$ into $m$ non-overlapping coalitions, $c_{1}, \ldots, c_{m}$, where $1 \leq m \leq a$. Let us define the set of coalitions resulting from such a partition as $C$, where $C=\left\{c_{1}, \ldots, c_{m}\right\}$ : $\forall i c_{i} \subseteq A ; \forall j: i \neq j, c_{i} \cap c_{j}=\emptyset ; \bigcup_{m} c_{i}=A$. Let us define the coalition ballot profile $K$ on $N \times C$, in terms of the ballot profile for alternatives, $V$. Let $K$ be the relation on $N \times A$ defined by $(i, c) \in K$, or $i K c$, if and only if $i$ votes for an alternative in coalition $c$. Thus $\forall i$ in $A, c$ in $C$ and $K$ in $N \times C, i K c \Leftrightarrow \exists j$ in $c: i V j$. Let us define the coalition vote total function for partition $C$ as the sum of the votes received by each alternative in the coalition. Thus $U: K \rightarrow[0, n]^{m} ; U_{k}=\sum_{i \in N} K_{k}=$ $\sum_{j \in c_{k}} \sum_{i \in N} V_{j}$, where $k=1, \ldots, m$. Let us define the coalition seat share function for partition $C$ as the sum of the seats allocated to each alternative in the coalition. Thus $F: K \rightarrow[0,1]^{m} ; F_{k}=\sum_{j \in c_{k}} E_{j}(V)$, where $k=1, \ldots, m$.

We can also redefine the properties used in Proposition 1 for use with coalitions. A seat allocation function $E$ is anonymous (neutral, nonnegativity responsive, canceling, weakly plurality ranking) for coalitions iff, for all partitions $C$, the coalition seat 
allocation function $F$ derived from it is anonymous (neutral, nonnegativity responsive, canceling, weakly plurality ranking). A coalition seat allocation function $F$ is anonymous iff $\forall K$ and $\sigma, F(K)=F(\sigma K)$, where $\sigma$ is a function that permutes $N$. It is neutral iff $\forall K$ and $\tau, \tau F(K)=F(\tau K)$, where $\tau$ is a function that permutes C. It satisfies the cancellation property iff $\forall V \forall j, k$ in $C, U_{j}=U_{k} \Rightarrow F_{j}=F_{k}$. (If the vote totals for two coalitions are the same, then their seat shares must be the same.) Let $R_{c}(K)$ be the set of voters that vote for an alternative in coalition $c$ under ballot profile $K$. Thus $R_{c}(K)=\{i: i K c\}$. F is nonnegativity responsive iff $\forall C \forall K, K^{\prime}$ in $N \times C$ and $c$ in $C, R_{c}\left(K^{\prime}\right) \supseteq R_{c}(K) \Rightarrow F_{c}\left(V^{\prime}\right) \geq F_{c}(V) . F$ satisfies the weak plurality ranking property iff $\forall V$ and $C \forall j, k$ in $C, U_{j}>U_{k} \Rightarrow F_{j} \geq F_{k}$. (If the alternatives in coalition $j$ win more votes than the alternatives in coalition $k$, coalition $j$ receives a greater or equal aggregate seat share to coalition $k$.).

Proposition 2 For any ballot profile $V$ and partition into coalitions $C$, any seat share allocation rule E that is anonymous, neutral and nonnegativity responsive for coalitions satisfies the weak plurality ranking property for coalitions. (Proof in Appendix)

The proof of this proposition is essentially identical to that of Proposition 1. If one coalition of alternatives receives more votes than another, it must receive at least an equal total seat share.

In representative bodies, governments are typically formed by a process of majority-rule coalition formation. Which coalition forms is a result of a bargaining process. However, the bargaining situation is defined in terms of which coalitions have sufficient seats to win a majority-rule vote of investiture (or confidence) and form a government. We can show that any seat allocation function that satisfies the coalitional weak plurality ranking property defines a coalition formation game identical to that defined by pure PR, except that ties between unevenly sized coalitions are sometimes possible. Therefore anonymity, neutrality and nonnegative responsiveness imply a seat share allocation that produces a parliamentary outcome almost identical to that produced by pure PR.

Formally, let us define pure PR as a seat share allocation function that allocates a seat share (a real number in $[0,1]$ ) to each list, which is equal to the share of the total vote that list won. Coalition games can be defined in terms of the set of winning coalitions $W \subseteq C$. Under majority rule, a coalition is winning iff it has a majority of seat share: $c \in W$ iff $F_{c}>F_{A-c}$. Under pure PR, a coalition wins a majority of seat share iff it has more than $50 \%$ of the vote: $c \in W$ iff $U_{c}>U_{A-c}$.

Proposition 3 For any ballot profile $V$ and partition into coalitions $C$, any seat share allocation function $E$ that is anonymous, neutral and nonnegativity responsive for coalitions defines a majority rule coalition game with a set of winning coalitions that is a subset of that defined by seat share allocation by proportionality. (Proof in Appendix)

The intuition behind the proof comes from the fact that under majority rule a coalition is winning if it has a greater seat share than all the alternatives excluded from it. By Proposition 2, anonymity, neutrality and nonnegative responsiveness require that if a coalition has more votes than another coalition, it must receive at least an equal 
seat share. Therefore the set of winning coalitions must be within the set of coalitions that have more votes than their complement (that is, have a majority of the vote). Thus it must be a subset of the set of winning coalitions under pure PR. Under these conditions, it is possible for a coalition to win a majority of the vote, but only to receive exactly half the seats, and thus be a blocking, but not winning coalition. However, it is impossible to have a "manufactured majority" (a situation where an alternative or coalition with a minority of the vote gets a majority of the seats) without violating anonymity or neutrality.

If we assume not just nonnegative responsiveness, but positive responsiveness then anonymity, neutrality and positive responsiveness imply a seat share allocation that produces a parliamentary outcome absolutely identical to that produced by pure PR (if a coalition wins a majority of the vote, it must get a majority of the seats). Seat share function $E$ is positively responsive iff it is nonnegativity responsive and $\forall V, V^{\prime}$ in $N \times A$ and $j$ in $A, S_{j}\left(V^{\prime}\right) \supset S_{j}(V) \Rightarrow E_{j}\left(V^{\prime}\right)>E_{j}(V)$. Similarly, coalition seat share function $F$ is positively responsive iff it is nonnegativity responsive and $\forall C, \forall K, K^{\prime}$ in $N \times C$ and $c$ in $C, R_{c}\left(K^{\prime}\right) \supset R_{c}(K) \Rightarrow F_{c}\left(V^{\prime}\right)>F_{c}(V)$.

Proposition 4 For any ballot profile and partition into coalitions, any seat share allocation function $E$ that is anonymous, neutral and positively responsive for coalitions defines a majority rule coalition game with a set of winning coalition that is identical to that defined by seat share allocation by proportionality. (Proof in Appendix)

\section{Discussion}

We have shown that any single-vote seat share allocation rule that satisfies anonymity, neutrality and nonnegativity responsiveness must produce results that are identical to those of pure PR, except that ties between unequally sized coalitions are possible. If we assume positive responsiveness, this exception is removed and the results must be identical to those under pure PR. Thus, PR can be justified in terms of liberal political equality and popular sovereignty, that is, in terms of the equal treatment of individual voters and the ability of the voters to make the final decision.

Pure proportionality is an ideal that can only be approximated by real world voting systems. This is because such systems have to allocate a finite number of seats, which can always produce some rounding error. Our results show that liberal political equality and popular sovereignty imply pure PR. What practical voting rule best approximates this ideal is beyond the scope of this paper (see Balinski and Young 1982/2003).

Our results only apply to single-vote seat allocation rules. Characterizing anonymous, neutral and nonnegativity responsive ordinal voting rules is beyond the scope of this paper. However, if an ordinal rule is to satisfy the requirement of liberal political equality, it must be compatible with proportionality in the special case where voters are only concerned about their preferred list. Suppose voters have list preferences (they all rank the candidates on one electoral list in the order of the list, and are indifferent between all the candidates not on that list). If this is the case, the preferences of every voter can be summarized by a ballot profile ("voter 1 supports list y"). If this is so, the results of the previous section can be applied to show that anonymity, neutrality 
and nonnegative responsiveness implies proportionality in this case. The point here is to provide a test of whether a particular ordinal system satisfies the value of liberal political equality. Clearly, ordinal systems give voters many more choices than simply voting for a straight list. However, if all voters were to have list preferences, and an ordinal system fails to deliver proportionality in this simple hypothetical case, then this ordinal system clearly violates liberal political equality.

This brings us to the limits of axiomatic theory. Few people would argue that liberal political equality is the only value to be considered in producing a "good" political system. Indeed, Schumpeter (1942) argued that it was not a value worth considering at all, and that democracy should be considered instrumental in terms of producing other values. Certainly we would have to balance the value of liberal political equality against values such as the protection of minority rights, stability and accountability. The question of how such liberal political equality and these other values can be balanced (if indeed there is a trade-off at all) is an empirical issue and is not pursued here. There is a considerable empirical literature on the political effects of electoral systems (Rae 1967; Taagepera and Shugart 1988; Lijphart 1994; Katz 1997; Farrell 2001 amongst others).

It is notable that single-member district plurality ("first-past-the-post") elections violate the liberal political equality of voters, not of parties. The axiom that is violated is anonymity, not neutrality. Both popular and academic debate about PR has tended to focus on arguments that PR is "fairer" because it treats all parties equally, while first-past-the-post discriminates against small and geographically dispersed parties. However, first-past-the-post is neutral, in that if all the voters for party A were to vote for party B and vice versa, the parties' seat totals would be reversed. The inequality in first-past-the-post elections comes from the fact that some people's votes count for more than others. However, the degree to which a vote counts does not depend on the individual characteristics of the voter, but on the distribution of opinion in the constituency in which that voter lives. If the distribution of opinion was random and unpredictable, this might be unproblematic; but this is clearly not the case. Thus the debate on PR could be more appropriately framed in terms of the rights of individual voters rather than in terms of fairness to political parties.

Open Access This article is distributed under the terms of the Creative Commons Attribution Noncommercial License which permits any noncommercial use, distribution, and reproduction in any medium, provided the original author(s) and source are credited.

\section{Appendix: Proofs}

Lemma 1 For any ballot profile V, any anonymous and neutral seat share allocation function satisfies the cancellation property.

The proof is isomorphic to the proof of Lemma 2 in Hout et al. (2002).

We need to show that $\forall V \forall j$ and $k$ in $A, T_{j}=T_{k} \Rightarrow E_{j}=E_{k}$ when anonymity and neutrality hold.

For any ballot profile $V$ where $T_{j}=T_{k}$, we can permute all voters so that the voters of alternatives $j$ and $k$ change places. We will call the permutation function $\sigma^{\prime}$. 
By anonymity, the seat shares stay the same: $E_{j}\left(\sigma^{\prime} V\right)=E_{j}(V), E_{k}\left(\sigma^{\prime} V\right)=$ $E_{k}(V)$.

We can permute the parties so that alternatives $j$ and $k$ change places. We call this permutation function $\pi^{\prime}$.

By neutrality, the seat share again remains the same: $E_{j}\left(\pi^{\prime} \sigma^{\prime} V\right)=E_{k}\left(\sigma^{\prime} V\right)$.

However, by construction $\pi^{\prime} \sigma^{\prime} V=V$. (We have swapped the supporters of alternatives $j$ and $k$, and then swapped the names of the parties, so we are back to the original situation.)

Therefore, $E_{j}\left(\pi^{\prime} \sigma^{\prime} V\right)=E_{j}(V)=E_{\mathrm{k}}\left(\sigma^{\prime} V\right)=E_{\mathrm{k}}(V)$.

Proposition 1 For any ballot profile $V$, any seat share allocation rule E that is anonymous, neutral and nonnegativity responsive satisfies the weak plurality ranking property.

The proof is isomorphic to the proof of Theorem 2 in Hout et al. (2002). We need to show that neutrality, anonymity and nonnegative responsiveness imply $\forall V \forall j, k$ in $A, T_{j}>T_{k} \Rightarrow E_{j} \geq E_{k}$.

By Lemma 1, anonymity and neutrality imply the cancellation property: $\forall V \forall j$ and $k$ in $A, T_{j}=T_{k} \Rightarrow E_{j}=E_{k}$.

Let $V^{\prime \prime}$ be a ballot profile where $T_{j}>T_{k}$.

Then we can derive a ballot profile $V^{\prime}$ where $T_{j}=T_{k}$ by having an appropriate number of voters for alternative $j$ abstain, so that the vote for alternative $j$ and $k$ are equal.

By the cancellation property: $E_{j}\left(V^{\prime}\right)=E_{k}\left(V^{\prime}\right)$.

By nonnegative responsiveness, $E_{j}\left(V^{\prime}\right)=E_{k}\left(V^{\prime}\right) \Rightarrow E_{j}\left(V^{\prime \prime}\right) \geq E_{k}\left(V^{\prime \prime}\right)$.

Lemma 2 For any ballot profile $V$ and partition into coalitions $C$, any anonymous and neutral coalition seat share allocation function $F$ satisfies the cancellation property.

Proof Isomorphic to Lemma 1.

Lemma 3 For any ballot profile $V$ and partition into coalitions $C$, any coalition seat share allocation rule $F$ that is anonymous, neutral and nonneagatively responsive satisfies the weak plurality ranking property.

Proof Isomorphic to Proposition 1.

Proposition 2 For any ballot profile $V$ and partition into coalitions $C$, any share allocation rule $E$ that is anonymous, neutral and nonnegativity responsive for coalitions satisfies the weak plurality ranking property for coalitions.

If seat share allocation function $E$ is anonymous, neutral and nonnegativity responsive for coalitions, then, for any partition $C$, by definition the coalition seat share function $F$ derived from it must be anonymous, neutral and nonnegativity responsive.

By Lemma 3, if $F$ is anonymous, neutral and nonnegativity responsive, it must satisfy the weak plurality ranking property.

If, for any partition $C, F$ satisfies the weak plurality ranking property, then by definition $E$ satisfies the weak plurality ranking property for coalitions. 
Proposition 3 For any ballot profile and partition into coalitions, any seat share allocation function $E$ that is anonymous, neutral and nonnegativity responsive for coalitions defines a majority rule coalition game with a set of winning coalition that is a subset of that defined by seat share allocation by proportionality.

Given that the set of majority rule winning coalitions is defined as $\left\{c \in C: F_{c}>\right.$ $\left.F_{A-c}\right\}$, and under pure PR the set of coalitions with a majority of seat share is $\left\{c \in C: U_{c}>U_{A-c}\right\}$, we need to show that if seat allocation function $E$ is anonymous, neutral and nonnegativity responsive for coalitions, then $\forall c$ in $C, U_{c}>U_{A-c} \Leftarrow$ $F_{c}>F_{A-c}$.

If the seat allocation function $E$ is anonymous, neutral and nonnegativity responsive for coalitions, then by Proposition 2 it satisfies the weak plurality ranking property for coalitions, and thus the coalition seat share allocation $F$ derived from it must satisfy the weak plurality ranking property: $\forall V$ and $C \forall j, k$ in $C, U_{j}>U_{k} \Rightarrow F_{j} \geq F_{k}$.

Suppose $F_{C}>F_{A-c}$, but $U_{c} \leq U_{A-c}$. If $U_{c}<U_{A-c}$ then by the weak plurality ranking property, $F_{c} \leq F_{A-c}$. Contradiction. If $E$ is anonymous and neutral for coalitions, then $F$ must be anonymous and neutral, and so by Lemma 2 must satisfy the cancellation property. If $U_{c}=U_{A-c}$, then by the cancellation property $F_{c}=F_{A-c}$. Contradiction.

Proposition 4 For any ballot profile and partition into coalitions, any seat share allocation function $E$ that is anonymous, neutral and positively responsive for coalitions defines a majority rule coalition game with a set of winning coalition that is identical to that defined by seat share allocation by proportionality.

If we substitute positive responsiveness for nonnegative responsiveness in the final line of the proof of Proposition 1, we show that any seat share allocation function $E$ that is anonymous, neutral and positively responsive satisfies the strong plurality ranking property: $\forall V \forall j$ and $k$ in $A, T_{j}>T_{k} \Rightarrow E_{j}>E_{k}$. Similarly, if we substitute positive responsiveness for negative responsiveness in Proposition 2, then any coalition seat share allocation function $F$ that is anonymous, neutral and positively responsive satisfies the strong plurality ranking property $: \forall V$ and $C \forall j, k$ in $C, U_{j}>U_{k} \Rightarrow F_{j}>F_{k}$

We need to show that if seat allocation function $E$ is anonymous, neutral and positively responsive for coalitions, then $\forall(c \in C) U_{c}>U_{A-c} \Leftrightarrow F_{c}>F_{A-c}$.

By Proposition 3, we have already shown that $\forall(c \in C) U_{c}>U_{A-c} \Leftarrow F_{c}>$ $F_{A-c}$.

All that remains is to show that $\forall(c \in C) U_{c}>U_{A-c} \Rightarrow F_{c}>F_{A-c}$, which follows directly from the strong plurality ranking property for coalitions.

\section{References}

Aumann R, Maschler M (1985) Game theoretic analysis of a distribution problem from the Talmud. J Econ Theory 36:195-213

Austen-Smith D, Banks J (2005) Positive political theory II. University of Michigan Press, Ann Arbor

Balinski M, Young H (1982) Fair representation: meeting the ideal of one man, one vote. Brookings Institute, Washington, DC

Banker R (1981) Equity considerations in traditional full cost allocation practices: an axiomatic approach. In: Moriarty S (ed) Joint cost allocations. University of Oklahoma Press, Oklahoma City 
Christiano T (1996) The rule of the many: fundamental issues in democratic theory. Westview Press, Boulder

Farrell D (2001) Electoral systems: a comparative introduction. Palgrave, Basingstoke

Hout E van der, de Swart H, ter Veer A (2002) Axioms characterizing the plurality ranking rule. Paper presented at the annual meeting of the Public Choice Society, San Diego, March 22-24 (submitted)

Independent Commission on the Voting System (1998) Report of the Independent Commission on the Voting System. The Stationary Office Cm 4090-1, London

Katz R (1997) Democracy and elections. Oxford University Press, Oxford

Lijphart A (1994) Electoral systems and party systems: a study of twenty-seven democracies, 1945-1990. Oxford University Press, Oxford

May K (1952) A set of independent necessary and sufficient conditions for simple majority decision. Econometrica 20:680-684

McLean I (1991) Forms of representation and voting systems. In: Held D (ed) Political theory today. Stanford University Press, Palo Alto

Moulin H (2002) The proportional random allocation of indivisible units. Soc Choice Welf 19(2):381-413

O'Neill B (1982) A problem of rights allocation from the Talmud. Math Soc Sci 2:345-371

Pitkin H (1967) The concept of representation. University of California Press, Berkeley

Powell G (2000) Elections as instruments of democracy: majoritarian and proportional visions. Yale University Press, New Haven

Rae D (1967) The political consequences of electoral laws. Yale University Press, New Haven

Rogowski R (1981) Representation in political theory and in law. Ethics 91(3):395-430

Saari D (2003) Capturing the 'Will of the People'. Ethics 113:333-349

Schumpeter J (1942) Capitalism, socialism and democracy. Harper, New York

Still J (1981) Liberal political equality and election systems. Ethics 91(3):375-394

Taagepera R, Shugart M (1988) Seats and votes: the effects and determinants of electoral systems. Yale University Press, New Haven

van Deemen AMA (1993) Paradoxes of voting in list systems of proportional representation. Elect Stud 12:234-241

Ward H (1995) A contractarian defense of ideal proportional representation. J Polit Philos 3(1):86-109

Young H (1974) An axiomatization of Borda's rule. J Econ Theory 9:43-52

Young H (1995) Optimal voting rules. J Econ Perspect 9(1):51-64 